\title{
Patients' Bedsides or Drug Trolleys: Where to Keep Paper Medication Administration Records?
}

\section{Eugene Y H Yeung}

Royal Lancaster Infirmary, Lancaster, UK

*Corresponding author: Eugene Y H Yeung, Education Centre, Royal Lancaster Infirmary, Ashton Road, Lancaster LA1 4RP, UK, Tel: 44(0)1524 65944; E-mail: eugeneyh@gmail.com

Received date: June 16, 2017; Accepted date: July 21, 2017; Published date: July 25, 2017

Copyright: ( 2017 , Yeung EYH. This is an open-access article distributed under the terms of the Creative Commons Attribution License, which permits unrestricted use, distribution, and reproduction in any medium, provided the original author and source are credited

\begin{abstract}
A point estimate study was conducted at a general teaching hospital in Britain that measured the availability of patients' paper medication administration records (also known as MARs or drug charts) in five surgical and two medical units. The five surgical units kept MARs at patients' bedsides, whereas each of the two medical units kept all MARs at two designated drug trolleys. MARs were readily available $76 \%$ ( 84 of 111 patients) of the time in the surgical units, compared to $94 \%$ (45 of 48 patients) of the time in the medical units. The difference in availability was statistically significant $(p<0.01)$. The results suggested that hospitals should keep paper MARs at a designated area rather than patients' bedsides. This setup would reduce the time needed to locate MARs, and hence make the work of doctors, nurses, and pharmacists more efficient.
\end{abstract}

Keywords: Nursing; Drug chart; Medication administration; Prescription; Medication error; Medication administration record; Hospital

\section{Introduction}

For at least the last 50 years, keeping paper medication administration records (also known as MARs or drug charts) at patients' bedsides appears to be the norm in British hospitals [1,2]. However, some wards may opt to keep all MARs at a designated area, such as drug trolleys, nursing stations, or treatment rooms. This setup facilitates drug preparation by nurses, who seldom prepare drugs at patients' bedsides. This also prevents MARs being misplaced and patients reading their own medication records [3].

\section{Methods}

In February 2017, a point estimate study was conducted at the Royal Lancaster Infirmary, a general teaching hospital in Britain. The study measured the availability of patients' paper MARs in five surgical and two medical units. The study obtained approval from the hospital audit department. The five surgical units kept MARs at patients' bedsides, whereas each of the two medical units kept all MARs at two designated drug trolleys. This hospital did not use electronic prescribing at the time of the study. Data were collected about the same time each day (16:00 to 17:30), but not during the drug rounds. Because there was no prior study data for calculation of sample size, all patients were included in the study to ensure adequate statistical power. Statistical analyses were performed using the software GraphPad Prism version 4.00 for Windows (GraphPad Software, San Diego, California, USA). Statistical significance was determined using two-sided Fisher's exact test and defined a priori as $\mathrm{p}<0.05$.

\section{Results and Discussion}

The study included all of the 159 patients in five surgical and two medical units. MARs were readily available $76 \%$ (84 of 111 patients) of the time in the surgical units, compared to $94 \%$ (45 of 48 patients) of the time in the medical units. The difference in availability was statistically significant $(\mathrm{p}<0.01)$. This study is limited by its small sample size and single-centred design. A potential confounder was the different work process in the surgical and medical units. However, the results suggested that hospitals should keep paper MARs at a designated area rather than patients' bedsides. This setup would reduce the time needed to locate MARs, and hence make the work of doctors, nurses, and pharmacists more efficient. When hospitals fully implement electronic prescribing, we may no longer have problems of searching for MARs [4,5]. Until then, we should make every effort to make paper MARs easily accessible, and not prone to being misplaced. Other advantages of electronic prescribing includes preventing errors in medication reconciliation and adverse drug reaction documentation, which frequently happen in hospitals $[6,7]$.

\section{Conclusion}

It can be concluded that, this study is another piece of evidence that supports full implementation of electronic prescribing in all hospitals.

\section{Conflict of Interest}

The author declared no other relationships or activities that could appear to have influenced the submitted work.

\section{References}

1. Crooks J, Clark CG, Caie HB, Mawson WB (1965) Prescribing and administration of drugs in hospital. Lancet 285: 373-378.

2. Barber N, Franklin BD, Jacklin A (2013) Of snarks, boojums and national drug charts. J R Soc Med 106: 6-8.

3. Garfield S, Jheeta S, Husson F, Lloyd J, Taylor A, et al.(2016) The role of hospital inpatients in supporting medication safety: A qualitative study. Plos one 11: e0153721. 
Citation: Yeung EYH (2017) Patients' Bedsides or Drug Trolleys: Where to Keep Paper Medication Administration Records? Gen Med (Los Angeles) 5: 292. doi:10.4172/2327-5146.1000292

Page 2 of 2

4. Van Wilder A, Bell H, Franklin BD (2016) The effect of electronic prescribing and medication administration on nurses' workflow and activities: An uncontrolled before and after study. Safety in Health 2: 13.

5. Coleman J (2013) Electronic solutions to safer hospital prescribing. Clin Ther 35: e114.
6. Yeung EY (2015) Adverse drug reactions: A potential role for pharmacists. Br J Gen Pract 65: 511.

7. Yeung EY (2016) Are we legitimately stopping medications? Use of pharmacist and junior doctor teaching to improve medication reconciliation. Br J Gen Pract. 